\title{
Deepwater fish assemblages at Isla del Coco National Park and Las Gemelas Seamount, Costa Rica
}

\author{
Richard M. Starr ${ }^{1}$, Kristen Green ${ }^{2}$ \& Enric Sala ${ }^{3,4}$ \\ 1. University of California Sea Grant Program and Moss Landing Marine Laboratories, 8272 Moss Landing Road, Moss \\ Landing, California 95039, USA; starr@mlml.calstate.edu \\ 2. Alaska Department of Fish and Game, 304 Lake Street, Room 103, Sitka, Alaska 99835; kristen.green@alaska.gov \\ 3. National Geographic Society, 20036 Washington, DC, USA; esala@ngs.org \\ 4. Centre d'Estudis Avançats de Blanes, CSIC, 17300 Blanes, Spain
}

Received 05-III-2012. Corrected 15-VI-2012. Accepted 24-IX-2012.

\begin{abstract}
The deepwater faunas of oceanic islands and seamounts of the Eastern Tropical Pacific are poorly known. From 11-22 September 2009 we conducted an exploration of the deepwater areas of the Isla del Coco Marine Conservation Area, Costa Rica and a nearby seamount using a manned submersible. The goal of the exploration was to characterize the habitats and biota, and conduct quantitative surveys of the deepwater portions of Isla del Coco National Park and Las Gemelas Seamount, located about 50km southwest of Isla del Coco. We completed a total of 22 submersible dives, spanning more than $80 \mathrm{hr}$ underwater, and collected a total of $36 \mathrm{hr}$ of video. We surveyed habitats from 50-402m and observed more than 45 species of fishes, some of which have not yet been described and are likely new to science. The diversity of fish species in deep water at Isla del Coco National Park was lower than the diversity of fishes in shallow water, and eight species groups accounted for more than $95 \%$ of the total fish biomass. The combined density of all fish species was higher at Las Gemelas Seamount $\left(253\right.$ fishes $\left./ 100 \mathrm{~m}^{2}\right)$ than at Isla del Coco National Park (138 fishes/100 $\left.\mathrm{m}^{2}\right)$. The combined density of fishes in habitats comprised primarily of bedrock or large boulders outcrops was more than three times as high at Las Gemelas Seamount as it was at Isla del Coco National Park. This discrepancy was caused by the extremely high concentration of Anthiinae fishes in rocky habitats at Las Gemelas Seamount. Densities of fishes in the other habitats were similar between the two sites. Similarly, when estimates of fish density were plotted by slope categories the density was much greater on steep slopes, which were usually comprised of rock habitats. Also, the density of fishes was greatest on high rugosity habitats. Results of these submersible surveys indicate that seamounts in the tropical eastern Pacific Ocean may be an important source of biodiversity and that more quantitative surveys are needed to characterize the fauna of the region. Citation: Starr, R.M., K. Green \& E. Sala. 2012. Deepwater fish assemblages at Coco Island National Park and Las Gemelas Seamounts, Costa Rica. Rev. Biol. Trop. 60 (Suppl. 3): 347-362. Epub 2012 Dec 01.
\end{abstract}

Key words: seamounts, deepwater habitats and fishes, submersible observations, biodiversity, census, fish assemblages.

Isla del Coco, also known as Cocos Island, is located $550 \mathrm{~km}$ southwest of the Pacific coast of Costa Rica and sits atop the Coco Volcanic Cordillera, a submarine mountain range that exists offshore of the southern part of Costa Rica (Cortés 2008, Alvarado 2009). The island is near the intersection of the Panama current and the northern equatorial counter-current. This convergence of large current systems creates localized currents that flow up the sides of the undersea ridge from the deep ocean, bringing cooler, nutrient rich water upward where it mixes with warmer surface waters to support an extremely productive ecosystem (Lizano 2008). The productive waters harbor a high diversity of fishes for the Eastern Tropical Pacific; more than 280 fishes have been described in waters less than $50 \mathrm{~m}$ deep (24 of which are endemic to Isla del Coco National Park) and more species, along with several 
unidentified species, have been seen in deeper waters (Garrison 2005, Cortés \& Blum 2008).

In recognition of the large diversity and uniqueness of flora and fauna associated with Isla del Coco, the government of Costa Rica designated Cocos Island a national park in 1978. As worldwide awareness of the rich environment increased, the United Nations Educational, Scientific and Cultural Organization (UNESCO) in 1997 declared Cocos Island a World Natural Heritage Site. A recent study reported that the average total biomass of reef fishes in the shallow waters around Cocos Island was $7.8 \mathrm{t} / \mathrm{ha}$, among the largest in the tropics worldwide (Friedlander et al. Revista de Biología Tropical, this issue). This makes Isla del Coco National Park a place of unique global value, and Costa Rica provided further protection to the region in 2001 by placing a $22.2 \mathrm{~km}$ no-fishing buffer surrounding the national park at Cocos Island.

In 2007, a conservation gap analysis was conducted, and Las Gemelas Seamount was identified as a possible location for inclusion into the system of marine reserves in Costa Rica (SINAC, MINAET 2008). Reports from Costa Rican fishers, however, indicated that this seamount has been fished occasionally in the last 15 years. In order to determine if the habitats and species of Las Gemelas Seamount were suitable for inclusion in a reserve system, we conducted submersible surveys to compare the seamount with the habitats and fauna of the deeper portions of waters around Isla del Coco National Park. We characterized the habitats and biota, and conducted quantitative surveys of the deepwater portions of Isla del Coco National Park and Las Gemelas Seamount. The objectives of the cruise with respect to fishes, were to gather quantitative information about species composition, numerical density, biomass, distribution and habitat associations of demersal fishes, and to compare the fish assemblages between Isla del Coco National Park and Las Gemelas Seamount.

\section{METHODS}

From 11-22 September 2009, scientists from the National Geographic Society, University of Costa Rica, Moss Landing Marine Laboratories, Monterey Bay Aquarium Research Institute, Ocean Research \& Conservation Association, and the University of California conducted an exploration of the deepwater areas around Isla del Coco National Park $\left(\sim 5^{\circ} 33^{\prime} \mathrm{N}, 87^{\circ} 02^{\prime} \mathrm{W}\right)$ and Las Gemelas Seamount $\left(\sim 4^{\circ} 59^{\prime} \mathrm{N}, 87^{\circ} 38^{\prime} \mathrm{W}\right)$, located about $50 \mathrm{~km}$ southwest of Isla del Coco National Park. We used the Undersea Hunter Group's DeepSee submersible to explore the water column and seafloor habitats to a depth of $400 \mathrm{~m}$ (Cortés \& Blum 2008).

At Las Gemelas Seamount, we surveyed a general area that was suggested to us by commercial fishermen. When we arrived in the general vicinity of the seamount, we conducted bathymetric surveys of the region using the support vessel's echosounder to locate the shallowest parts of the seamount, and then used the DeepSee submersible to survey two of the shallow peaks. At Isla del Coco National Park, most submersible dives occurred at dive locations along the drop-off at the northern edge of the island, in areas normally visited by the DeepSee submersible during its regular trips with commercial passengers. At all dive sites, observers usually spent $30 \mathrm{~min}$ to one hour exploring the area. After getting a sense of the habitat types associated with the dive site, observers haphazardly chose starting depths and directions for quantitative surveys. Observers most frequently chose to start a transect at a habitat and depth representative of the site (often a rock outcrop). Upon starting a transect, the pilot would slowly $(\sim 0.15 \mathrm{~m} / \mathrm{sec})$ drive the submersible along a preselected course for $10 \mathrm{~min}$. The course was almost always either parallel to an isobath or angled about 45 degrees up or down to the isobath. On one transect, the submersible transect was nearly 
vertical. During a typical $3 \mathrm{hr}$-long dive, we conducted two to four quantitative, 10min transects. The number and starting location of transects were selected by the scientific observer in the submersible.

Submersible transects were patterned after strip transect surveys that have been commonly used to evaluate fishes in temperate environments (Stein et al. 1992, Starr et al. 1996, Yoklavich et al. 2002). During these quantitative transects, observers looked forward and downward through the submersible dome, and recorded species composition, species-habitat associations, length distributions of fishes, and relative abundances and depth distributions of fishes. We identified and counted every fish observed in a swath that was $1 \mathrm{~m}$ wide, for a set time period (usually $10 \mathrm{~min}$ ). Lasers that were mounted $33 \mathrm{~cm}$ apart, on either side of the camera housing, shined parallel beams of light and allowed us to establish transect width. Pilots maneuvered the submersible and/or adjusted the camera so that the camera's field of view was as close to $1 \mathrm{~m}$ wide as possible. Observers in the submersible used the paired lasers as a reference for scale and identified fishes within the $1 \mathrm{~m}$ strip transect. The lasers were also used to estimate the lengths of fishes observed on and off transect. Transect lengths were determined by distance traveled as measured by a Doppler velocity log attached to the submersible. In addition to direct observations, a video record of the transect swath was recorded by the submersible's high-definition digital camera on mini-DV tape. All video tapes were reviewed to record fishes missed by observers, verify the identification of species, describe and classify habitats, and verify that the observer only counted fishes within the transect width. We also recorded diver observations using a digital voice recorder.

Habitat data: Habitats were defined by a combination of substrate type and slope and rugosity of the seafloor, as described in Greene et al. (1999) and Tissot et al. (2007). We used seven primary substrate codes: boulder (B), cobble $(C)$, gravel $(G)$, pebble $(P)$, bedrock or rock outcrop (R), sand (S), and a code for a vertical pinnacle $(\mathrm{T})$. We defined bottom type as a two-letter code representing the approximate percent cover of the two most prevalent substrata in a particular habitat patch. The first character of the code represents the substratum that accounted for at least $50 \%$ of the habitat, and the second represents the second most prevalent habitat, accounting for at least $20 \%$ of the patch (e.g., the code RB represents a habitat in which at least $50 \%$ is bedrock and at least $20 \%$ of the bottom is covered by boulders). If bottom is entirely a single type of substrate, then we use a single code twice (e.g., "BB" for $>70 \%$ cover by boulders). Also, we defined three types of slope $\left(<30^{\circ}, 30-60^{\circ}\right.$, $>60^{\circ}$ ) and three rugosity levels (Low, Medium, High) that were arbitrarily defined, based on the capability of the crevices in the substrate to hide fishes (e.g., no hiding places, can hide small fishes, crevices large enough to hide fishes $>25 \mathrm{~cm}$ long). These approaches to habitat classification and analysis have long been used in sub-tropical submersible surveys (e.g., Pearcy et al. 1989, Stein et al. 1992, Starr et al. 1996, Greene et al. 1999, Yoklavich et al. 2002, Tissot et al. 2007, Starr \& Yoklavich 2008)

Analyses: We used only data from submersible dives that occurred at similar depths and covered similar habitats at each site to compare fish assemblages at Las Gemelas Seamount with those around Isla del Coco National Park. The dives and associated transects at Las Gemelas Seamount covered primarily rocky habitats at depths greater than $150 \mathrm{~m}$. Thus, for comparison purposes, we used only the nine submersible dives at Isla del Coco National Park that contained transects covering similar depths and habitats to contrast with the four submersible surveys at Las Gemelas Seamount. The comparisons included species composition, density in terms of numbers of fish and biomass (i.e., standing stock), and size composition of species or taxonomic group. We also recorded the number of times submersible observers saw fishing gear on transects. 
To evaluate species composition, we calculated species richness (the number of species) and diversity (Shannon-Wiener Index, Zar 1999) at each site. We then calculated species density for each transect by summing the number of fish observed on each transect or counted on the videotapes and dividing that number by the area of that transect (i.e., transect length $x$ $1 \mathrm{~m}$ width). Transect densities were averaged to provide an overall estimate of density for each taxonomic group. We then estimated biomass for taxonomic groups at each site by converting fish length to biomass, using length-weight relationships obtained from Fishbase (Froese \& Pauly 2012). When a conversion was not available for a particular species, we used a conversion factor from a similar species. Biomass was calculated for each transect and transects were averaged at each site to provide an estimate of standing stock (biomass per unit area). Finally, we estimated mean sizes of each taxa and evaluated size frequency distributions at each site and used a t-test to compare means.

\section{RESULTS}

Submersible dives and transects: We completed a total of 22 submersible dives. During the cruise, rough weather limited our opportunity to launch the submersible at Las Gemelas Seamount, and we were only able to conduct surveys there on only two days. We were able to launch the submersible on 12 days at Isla del Coco National Park. We conducted a total of four dives at the Las Gemelas Seamount and 18 dives around Isla del Coco National Park. Maximum depths of dives ranged from $50-402 \mathrm{~m}$, and dive duration averaged $3.7 \mathrm{hr}$. Total duration of visual observations during dive explorations was more than $80 \mathrm{hr}$.

Quantitative data were available from 16 submersible dives (Table 1). Four of these dives occurred at Las Gemelas Seamount, and 12 dives occurred around Isla del Coco National Park. A total of 38 quantitative transects were completed in this study. Transect lengths varied from $23-169 \mathrm{~m}$. The total area surveyed equaled $3003 \mathrm{~m}^{2}$. At Isla del Coco National
Park, we conducted 25 quantitative transects on 12 dives, and surveyed $1999 \mathrm{~m}^{2}$. At Las Gemelas Seamount, we completed 13 video transects on four dives, and surveyed $1004 \mathrm{~m}^{2}$.

We collected $36 \mathrm{hr}$ of video documentation of habitats, fishes, and macroinvertebrates. After the cruise we viewed the videotapes to identify species, species-habitat associations, length distributions of fishes, relative abundances and depth distributions of fishes, and potentially new species or those that are unreported from this region. To gather more information about species composition and length frequencies of fishes, we evaluated video from the parts of the submersible dives that were not on transect as well as the areas on transects.

Habitat data: We encountered a total of 18 combinations of the seven substratum codes. At Isla del Coco National Park, 58\% of the habitats surveyed were comprised primarily of rock and 34\% were primarily sand, whereas at Las Gemelas, $73 \%$ of the transects covered rocky habitats and $25 \%$ covered sandy habitats (Tables 2,3). Transects at Las Gemelas Seamount occurred over somewhat more rugose habitats. At Isla del Coco National Park, $39.9 \%, 28.5 \%$, and $31.6 \%$ of the habitat area surveyed was high, medium, and low rugosity, respectively. At Las Gemelas Seamount, $46.8 \%, 34.3 \%$, and $18.9 \%$ of the habitat area surveyed was high, medium, and low rugosity, respectively. Similarly, transects at Las Gemelas more typically occurred on higher slopes. At Las Gemelas Seamount, 30\%, 56\%, and $14 \%$ of the habitat area surveyed contained slopes of $<30^{\circ}, 30-60^{\circ}$, and $>60^{\circ}$, respectively, whereas at Isla del Coco National Park, it was $48 \%, 35 \%$, and $17 \%$.

Species composition: A total of 4,544 fishes were observed from 46 taxa on the quantitative transects that occurred at depths below $50 \mathrm{~m}$ (Table 4). All the species observed on transects above $50 \mathrm{~m}$ have been described in Garrison (2005), and are not reported here because only a few submersible transects were conducted in shallow water. In deeper waters, 
TABLE 1

Transect number (Tran. No.), number fish observed (No. Fish), transect distance (Distance), transect start and end depths, and change in transect depth (Depth Diff.), and location of submersible dives

\begin{tabular}{|c|c|c|c|c|c|c|c|}
\hline $\begin{array}{l}\text { Dive } \\
\text { No. }\end{array}$ & $\begin{array}{l}\text { Tran. } \\
\text { No }\end{array}$ & $\begin{array}{l}\text { No. } \\
\text { Fish }\end{array}$ & $\begin{array}{l}\text { Distance } \\
\text { (m) }\end{array}$ & $\begin{array}{l}\text { Start Depth } \\
\text { (m) }\end{array}$ & $\begin{array}{l}\text { End Depth } \\
\text { (m) }\end{array}$ & $\begin{array}{l}\text { Depth Diff. } \\
\text { (m) }\end{array}$ & Location \\
\hline 911 & 2 & 106 & 31 & 249 & 209 & -40 & Isla del Coco \\
\hline 911 & 3 & 69 & 70 & 199 & 175 & -24 & Isla del Coco \\
\hline 911 & 4 & 76 & 33 & 174 & 163 & -11 & Isla del Coco \\
\hline 912 & 2 & 19 & 96 & 257 & 245 & -12 & Isla del Coco \\
\hline 914 & 1 & 2 & 83 & 298 & 311 & 13 & Las Gemelas \\
\hline 914 & 2 & 67 & 63 & 302 & 283 & -19 & Las Gemelas \\
\hline 914 & 3 & 335 & 55 & 238 & 214 & -24 & Las Gemelas \\
\hline 915 & 1 & 80 & 69 & 249 & 230 & -19 & Las Gemelas \\
\hline 915 & 2 & 121 & 81 & 226 & 227 & 0 & Las Gemelas \\
\hline 915 & 3 & 145 & 61 & 226 & 214 & -12 & Las Gemelas \\
\hline 915 & 4 & 40 & 45 & 219 & 217 & -2 & Las Gemelas \\
\hline 916 & 1 & 8 & 100 & 290 & 300 & 10 & Las Gemelas \\
\hline 916 & 2 & 32 & 91 & 282 & 265 & -17 & Las Gemelas \\
\hline 916 & 3 & 321 & 77 & 255 & 227 & -28 & Las Gemelas \\
\hline 917 & 1 & 411 & 118 & 266 & 227 & -39 & Las Gemelas \\
\hline 917 & 2 & 702 & 76 & 221 & 200 & -21 & Las Gemelas \\
\hline 917 & 3 & 274 & 85 & 196 & 188 & -8 & Las Gemelas \\
\hline 918 & 1 & 0 & 107 & 375 & 393 & 18 & Isla del Coco \\
\hline 918 & 2 & 1 & 75 & 401 & 394 & -7 & Isla del Coco \\
\hline 919 & 1 & 16 & 112 & 106 & 104 & -3 & Isla del Coco \\
\hline 919 & 2 & 28 & 121 & 104 & 104 & -1 & Isla del Coco \\
\hline 920 & 1 & 34 & 62 & 225 & 218 & -7 & Isla del Coco \\
\hline 920 & 2 & 27 & 50 & 206 & 202 & -4 & Isla del Coco \\
\hline 920 & 3 & 60 & 90 & 201 & 222 & 21 & Isla del Coco \\
\hline 925 & 1 & 279 & 52 & 175 & 175 & 0 & Isla del Coco \\
\hline 925 & 2 & 476 & 96 & 175 & 180 & 5 & Isla del Coco \\
\hline 926 & 1 & 128 & 77 & 174 & 170 & -4 & Isla del Coco \\
\hline 926 & 2 & 57 & 77 & 154 & 156 & 2 & Isla del Coco \\
\hline 927 & 1 & 59 & 86 & 91 & 91 & 0 & Isla del Coco \\
\hline 928 & 1 & 22 & 63 & 70 & 77 & 7 & Isla del Coco \\
\hline 928 & 2 & 27 & 102 & 78 & 82 & 4 & Isla del Coco \\
\hline 928 & 3 & 56 & 73 & 82 & 43 & -39 & Isla del Coco \\
\hline 929 & 1 & 55 & 24 & 251 & 228 & -23 & Isla del Coco \\
\hline 929 & 2 & 30 & 32 & 222 & 192 & -30 & Isla del Coco \\
\hline 929 & 3 & 42 & 138 & 187 & 170 & -17 & Isla del Coco \\
\hline 930 & 1 & 11 & 67 & 72 & 62 & -10 & Isla del Coco \\
\hline 932 & 1 & 195 & 49 & 255 & 230 & -25 & Isla del Coco \\
\hline 932 & 3 & 40 & 48 & 229 & 223 & -6 & Isla del Coco \\
\hline 932 & 4 & 93 & 169 & 222 & 180 & -42 & Isla del Coco \\
\hline
\end{tabular}


TABLE 2

Area of different habitats surveyed on submersible dives completed at Las Gemelas Seamount. Habitat codes are based on the percentage of bottom covered by boulder (B), cobble (C), gravel (G), pebble (P), bedrock or rock outcrop (R), sand (S), or vertical pinnacle (T). The first letter represents the substrate type that accounted for at least $50 \%$ of the seafloor; the second code represents the substrate type that accounted for at least $20 \%$ of the seafloor. Rugosity levels

(H: High, M: Medium, L: Low) are based on the capability of the crevices in the substrate to hide fishes

\begin{tabular}{|c|c|c|c|c|c|c|c|c|c|c|c|}
\hline \multirow{3}{*}{$\begin{array}{l}\text { Habitat } \\
\text { Code }\end{array}$} & \multirow{2}{*}{\multicolumn{3}{|c|}{$\begin{array}{c}<30^{\circ} \text { Slope } \\
\text { Rugosity }\end{array}$}} & \multirow{2}{*}{\multicolumn{3}{|c|}{$\begin{array}{c}30-60^{\circ} \text { Slope } \\
\text { Rugosity }\end{array}$}} & \multirow{2}{*}{\multicolumn{3}{|c|}{$\begin{array}{c}>60^{\circ} \text { Slope } \\
\text { Rugosity }\end{array}$}} & \multirow{3}{*}{$\begin{array}{c}\text { Total } \\
\text { Area }\left(\mathrm{m}^{2}\right)\end{array}$} & \multirow{3}{*}{$\begin{array}{c}\text { Percent } \\
\text { Area }\end{array}$} \\
\hline & & & & & & & & & & & \\
\hline & $\mathrm{H}$ & M & $\mathrm{L}$ & $\mathrm{H}$ & M & $\mathrm{L}$ & $\mathrm{H}$ & M & $\mathrm{L}$ & & \\
\hline BB & 9.8 & & & 45.0 & 3.3 & & & & & 58.1 & 5.8 \\
\hline $\mathrm{BC}$ & & & & & & & & & & 0.0 & 0.0 \\
\hline BS & 7.1 & 7.1 & & 1.6 & 44.6 & & & & & 60.4 & 6.0 \\
\hline $\mathrm{CC}$ & & & & & & & & & & 0.0 & 0.0 \\
\hline CG & & & & & & & & & & 0.0 & 0.0 \\
\hline $\mathrm{CS}$ & & 11.4 & & & & & & & & 11.4 & 1.1 \\
\hline PC & & & & & & & & & & 0.0 & 0.0 \\
\hline PG & & & & & & & & & & 0.0 & 0.0 \\
\hline PS & & 8.6 & & & & & & & & 8.6 & 0.9 \\
\hline $\mathrm{RR}$ & 12.5 & 19.5 & & 171.4 & 72.9 & 1.9 & 70.9 & & & 349.1 & 34.8 \\
\hline RS & & 17.0 & & 2.8 & 80.2 & 16.2 & & & & 116.2 & 11.6 \\
\hline SB & & 66.7 & 1.5 & & 6.9 & 5.6 & & & & 80.8 & 8.0 \\
\hline $\mathrm{SC}$ & & 3.1 & 9.3 & & & & & & & 12.4 & 1.2 \\
\hline SG & & & 26.8 & & & 16.2 & & & & 42.9 & 4.3 \\
\hline SP & & 1.6 & 91.7 & & & 2.0 & & & & 95.4 & 9.5 \\
\hline SR & & & 7.9 & & 1.2 & 9.2 & & & & 18.2 & 1.8 \\
\hline SS & & & 1.9 & & & & & & & 1.9 & 0.2 \\
\hline TT & & & & 84.3 & & & 64.2 & & & 148.5 & 14.8 \\
\hline Sum & 29.4 & 135.0 & 139.0 & 305.1 & 209.1 & 51.1 & 135.1 & 0.0 & 0.0 & 1003.8 & \\
\hline
\end{tabular}

observers encountered unfamiliar species, and we were unable to resolve species identification despite sending photos to ichthyologists who are experts in different genera of fishes. To resolve this issue, species have been placed into taxonomic groups to make it easier to compare the fauna of Las Gemelas Seamount with the fauna at Isla del Coco National Park (Table 5). We know that some of the fishes we saw are either new species or are species that have not been reported for this region. For example, in addition to Pontinus clemensi and Scorpaenodes rubrivinctus (recently described by Poss et al. 2010), we saw at least six other species of Scorpionfish (Scorpaenidae). Based on discussions with taxonomists, some of the unidentified species are likely to be Pontinus strigatus (identified from the Galapagos Islands by Grove \& Lavenberg
(1997)), Scorpaena afuerae (identified from Ecuador by Jiménez \& Béarez (2004)), Pontinus furcirhinus (described by Poss (1995)), and two of the species we observed had characteristics similar to Trachyscorpia osheri and Idiastion hageyi, new species described from the Galapagos Islands by McCosker (2008). It was not possible, however, to identify to the species level all Scorpaenids recorded on video. Similarly, we observed two distinct species of Batfish (Ogcocephalidae), two species of Frogfish (Antennariidae), two species of Wrasse (Labridae) and two species of Conger Eel (Anguilliformes) that we could not identify to species using only photographs. These fishes may be the same as some of those described from the Galapagos (Grove and Lavenberg 1997) or they may be new, undescribed species. Voucher specimens are needed to determine 
TABLE 3

Area of different habitats surveyed on submersible dives completed at Isla del Coco National Park. Habitat codes are based on the percentage of bottom covered by boulder (B), cobble $(\mathrm{C})$, gravel $(\mathrm{G})$, pebble $(\mathrm{P})$, bedrock or rock outcrop $(\mathrm{R})$, sand $(\mathrm{S})$, or vertical pinnacle $(\mathrm{T})$. The first letter represents the substrate type that accounted for at least $50 \%$ of the seafloor; the second code represents the substrate type that accounted for at least $20 \%$ of the seafloor. Rugosity levels

(H: High, M: Medium, L: Low) are based on the capability of the crevices in the substrate to hide fishes

\begin{tabular}{|c|c|c|c|c|c|c|c|c|c|c|c|}
\hline \multirow{3}{*}{ Habitat } & \multicolumn{3}{|c|}{$<30^{\circ}$ Slope } & \multicolumn{3}{|c|}{$30-60^{\circ}$ Slope } & \multicolumn{3}{|c|}{$>60^{\circ}$ Slope } & \multirow{3}{*}{$\begin{array}{c}\text { Total Area } \\
\left(\mathrm{m}^{2}\right)\end{array}$} & \multirow{3}{*}{$\begin{array}{c}\% \\
\text { Area }\end{array}$} \\
\hline & \multicolumn{3}{|c|}{ Rugosity } & \multicolumn{3}{|c|}{ Rugosity } & \multicolumn{3}{|c|}{ Rugosity } & & \\
\hline & $\mathrm{H}$ & M & L & $\mathrm{H}$ & M & $\mathrm{L}$ & $\mathrm{H}$ & M & $\mathrm{L}$ & & \\
\hline BB & 23.2 & 7.3 & & & & & & & & 30.5 & 1.4 \\
\hline $\mathrm{BC}$ & & 4.5 & & & & & & & & 4.5 & 0.2 \\
\hline BS & 26.7 & 16.2 & & & & & & & & 42.9 & 2.0 \\
\hline $\mathrm{CC}$ & & 18.6 & & & & & & & & 18.6 & 0.9 \\
\hline CG & & & & & & & & & & 0.0 & 0.0 \\
\hline CS & & 62.8 & & & & 1.6 & & & 2.4 & 66.8 & 3.1 \\
\hline PC & & & & & & & & & & 0.0 & 0.0 \\
\hline PG & & & & & & & & & & 0.0 & 0.0 \\
\hline PS & & 2.1 & & & & 3.5 & & & & 5.6 & 0.3 \\
\hline $\mathrm{RR}$ & 88.9 & 26.4 & & 259.7 & 96.5 & & 213.5 & 12.7 & & 697.6 & 32.7 \\
\hline RS & 11.6 & 141.1 & & 105.2 & 148.1 & & 6.6 & 9.6 & & 422.3 & 19.8 \\
\hline SB & & 2.9 & & & & & & & & 2.9 & 0.1 \\
\hline $\mathrm{SC}$ & & 3.3 & & & & 9.0 & & & 3.0 & 15.3 & 0.7 \\
\hline SG & & & 98.0 & & & & & & & 98.0 & 4.6 \\
\hline SP & & & 58.2 & & & 22.6 & & & & 80.8 & 3.8 \\
\hline SR & & 22.1 & 2.4 & & 27.2 & 2.4 & & 3.3 & & 57.5 & 2.7 \\
\hline SS & & & 397.9 & & & 72.5 & & & 0.8 & 471.2 & 22.1 \\
\hline TT & & & & & & & 115.9 & 4.0 & & 119.9 & 5.6 \\
\hline Sum & 150.3 & 307.3 & 556.5 & 364.9 & 271.8 & 111.6 & 336.1 & 29.6 & 6.2 & 2134 & \\
\hline
\end{tabular}

whether or not these fishes are new species. Similarly, we observed several different types of Anthiinae species. Some of the fishes we saw were clearly Anthias noeli (Anderson \& Baldwin 2000, Béarez \& Jiménez Prado 2003) and some were clearly Pronotogrammus eos or P. multifasciatus. However, we saw at least two other types of fishes that were either different Anthiinae species or different morphological versions of the Anthiinae species we observed. These four or more species of Anthiids were usually in mixed aggregations on boulder or rocky habitats with medium-sized crevices.

Due primarily to the larger number of transects occurring in Isla del Coco National Park, species richness was greater there than at Las Gemelas Seamount. We encountered 28 taxa on quantitative transects at Isla del Coco National Park and 16 taxa at Las Gemelas. Because we encountered several fishes that have not yet been reported in the scientific literature, we grouped species into higher taxonomic levels for our analyses. Flagfins (Aulopidae), scorpionfishes (Scorpaenidae), and serranids (Serranidae) were the dominant species groups at each site (Table 5). Flagfins were relatively common at Isla del Coco National Park, but this taxon was absent from the Las Gemelas dives. The diversity index ( $\left.\mathrm{H}^{\prime}\right)$ calculated for Isla del Coco National Park was 1.79 and species evenness (J) was 0.54. At Las Gemelas, the Shannon diversity index equaled 0.66 and species evenness was 0.24 . These values include only species on quantitative transects and assumes that the several different morphological versions of the Anthiinae species we observed are only one species.

Fish density and biomass: The combined density of all fish species was higher 
TABLE 4

Species groups observed on at Las Gemelas Seamount and Isla del Coco National Park

\begin{tabular}{|c|c|c|c|}
\hline Scientific Name & Common name & Las Gemelas $>50 \mathrm{~m}$ & Isla del Coco $>50 \mathrm{~m}$ \\
\hline Anguilliformes, unidentified & Conger eel sp. A & $\mathrm{X}$ & $\mathrm{X}$ \\
\hline Anguilliformes, unidentified & Conger eel sp. B & & $\mathrm{X}$ \\
\hline Antennarius avalonis & Frogfish & $\mathrm{X}$ & $\mathrm{X}$ \\
\hline Antennariidae & Frogfish sp. B & $\mathrm{X}$ & $\mathrm{X}$ \\
\hline Antennariidae & Frogfish sp. C & $\mathrm{X}$ & $\mathrm{X}$ \\
\hline Anthias noeli & Rosy jewelfish & $\mathrm{X}$ & $\mathrm{X}$ \\
\hline Anthiinae, unidentified & Frogfish & $\mathrm{X}$ & $\mathrm{X}$ \\
\hline Aulopus sp. & Eastern Pacific flagfin & & $\mathrm{X}$ \\
\hline Bathycongrus varidens & Conger eel & $\mathrm{X}$ & $\mathrm{X}$ \\
\hline Bellator loxias & Searobin & & $\mathrm{X}$ \\
\hline Brotula ordwayi & Speckled bearded cusk-eel & & $\mathrm{X}$ \\
\hline Brotula sp. & Cusk-eel & $\mathrm{X}$ & $\mathrm{X}$ \\
\hline Carcharhinus falciformis & Silky shark & & $\mathrm{X}$ \\
\hline Chlorophthalmus mento & Greeneyes & $\mathrm{X}$ & $\mathrm{X}$ \\
\hline Decodon melasma & Blackspot wrasse & & $\mathrm{X}$ \\
\hline Dibranchus crascens & Batfish & $\mathrm{X}$ & \\
\hline Epinephelus cifuentesi & Olive grouper & $\mathrm{X}$ & $\mathrm{X}$ \\
\hline Epinephelus niphobles & Snowy grouper & $X$ & $\mathrm{X}$ \\
\hline Guentherus altivela & Jellynose & $\mathrm{X}$ & $\mathrm{X}$ \\
\hline Kyphosidae & Chub & & $\mathrm{X}$ \\
\hline Labridae, unidentified & Wrasse sp. A & $\mathrm{X}$ & $\mathrm{X}$ \\
\hline Labridae, unidentified & Wrasse sp. B & & $\mathrm{X}$ \\
\hline Laemonema sp. & Cod & $\mathrm{X}$ & $\mathrm{X}$ \\
\hline Lophiodons spiluris & Goosefish & $\mathrm{X}$ & $\mathrm{X}$ \\
\hline Mobula tarapacana & Mobulid ray & & $\mathrm{X}$ \\
\hline Mycteroperca olfax & Sailfin grouper & & $\mathrm{X}$ \\
\hline Myrichthys tigrinus & Tiger snake eel & & $\mathrm{X}$ \\
\hline Myroconger nigrodantatus & Punch banana eel & $\mathrm{X}$ & $\mathrm{X}$ \\
\hline Ogcocephalidae & Batfish sp. A & $\mathrm{X}$ & \\
\hline Ogcocephalidae & Batfish sp. B & $\mathrm{X}$ & \\
\hline Ophidiidae & Cusk-eel & & $\mathrm{X}$ \\
\hline Opistognathidae & Jawfishes & & $\mathrm{X}$ \\
\hline Peristedion crustosum & Cocos Searobin & & $\mathrm{X}$ \\
\hline Physiculus sp. & Cod & $\mathrm{X}$ & $\mathrm{X}$ \\
\hline Pleuronectiformes, unidentified & Flatfish & & $\mathrm{X}$ \\
\hline Pontinus clemensi & Mottled scorpionfish & & $\mathrm{X}$ \\
\hline Pontinus sp. A (P. furcirhinus?) & Scorpionfish & $\mathrm{X}$ & \\
\hline Pontinus sp. B (P. strigatus?) & Scorpionfish & $\mathrm{X}$ & \\
\hline Pronotogrammus eos & Bigeye bass & $\mathrm{X}$ & $\mathrm{X}$ \\
\hline Pronotogrammus multifasciatus & Threadfin bass & $\mathrm{X}$ & $\mathrm{X}$ \\
\hline Remora remora & Remora & $\mathrm{X}$ & $\mathrm{X}$ \\
\hline Scorpaena sp. A (S. afuerae?) & Scorpionfish & & $\mathrm{X}$ \\
\hline Scorpaenidae, unidentified & Scorpionfish sp. A & $\mathrm{X}$ & \\
\hline Scorpaenidae, unidentified & Scorpionfish sp. B & $\mathrm{X}$ & $\mathrm{X}$ \\
\hline Scorpaenodes rubrivinctus & Scorpionfish & $\mathrm{X}$ & $\mathrm{X}$ \\
\hline Sphyrna lewini & Hammerhead shark & & $\mathrm{X}$ \\
\hline Total taxa & & 28 & 40 \\
\hline
\end{tabular}


TABLE 5

Species and taxonomic groups of fishes observed during quantitative transects at Isla del Coco National Park and Las Gemelas Seamount. The Number of Fish (\# Fish), Density (\# fish $\left./ 100 \mathrm{~m}^{2}\right)$, and Biomass $\left(\mathrm{kg} / 100 \mathrm{~m}^{2}\right)$ are reported for each location. The Biomass ratio is the biomass of each species $\left(\mathrm{kg} / 100 \mathrm{~m}^{2}\right)$ observed at Las Gemelas Seamount divided by the biomass of each species at Isla del Coco National Park

\begin{tabular}{|c|c|c|c|c|c|c|c|}
\hline \multirow[b]{2}{*}{ Common Name } & \multicolumn{3}{|c|}{ Isla del Coco National Park } & \multicolumn{3}{|c|}{ Las GemelasSeamount } & \multirow[b]{2}{*}{ Biomass Ratio } \\
\hline & \# Fish & $\begin{array}{l}\text { Density } \\
\left(\# / 100 \mathrm{~m}^{2}\right)\end{array}$ & $\begin{array}{c}\text { Biomass } \\
\left(\mathrm{kg} / 100 \mathrm{~m}^{2}\right)\end{array}$ & \# Fish & $\begin{array}{c}\text { Density } \\
\left(\# / 100 \mathrm{~m}^{2}\right)\end{array}$ & $\begin{array}{c}\text { Biomass } \\
\left(\mathrm{kg} / 100 \mathrm{~m}^{2}\right)\end{array}$ & \\
\hline Brotulas & 2 & 0.2 & 0.03 & & & & \\
\hline Codlings & 30 & 2.3 & 0.98 & 7 & 0.7 & 0.19 & 0.19 \\
\hline Eels & 5 & 0.4 & 0.03 & 32 & 3.2 & 0.18 & 5.98 \\
\hline Fish, unidentified & 83 & 6.5 & N/A & 54 & 5.4 & N/A & \\
\hline Flagfins & 203 & 15.9 & 5.26 & & & & \\
\hline Goosefishes & & & & 1 & 0.1 & 0.01 & \\
\hline Jellynose fish & 1 & 0.1 & 0.01 & & & & \\
\hline Ocean whitefish & 9 & 0.7 & 1.73 & & & & \\
\hline Scorpionfishes & 126 & 9.9 & 0.90 & 34 & 3.4 & 0.96 & 1.07 \\
\hline \multicolumn{8}{|l|}{ Serranids } \\
\hline Basslets & 422 & 33.0 & 2.10 & 1995 & 198.9 & 21.60 & 10.26 \\
\hline Groupers & 7 & 0.5 & 4.94 & 3 & 0.3 & 5.42 & 1.10 \\
\hline Threadfin Bass & 872 & 68.2 & 10.18 & 407 & 40.6 & 5.08 & 0.50 \\
\hline Wrasses & 9 & 0.7 & 2.12 & 5 & 0.5 & 0.06 & 0.03 \\
\hline Total & 1769 & 138 & 28 & 2538 & 253 & 33 & 1.2 \\
\hline
\end{tabular}

at Las Gemelas Seamount (253 fishes $/ 100 \mathrm{~m}^{2}$ ) than at Isla del Coco National Park (138 fishes $\left./ 100 \mathrm{~m}^{2}\right)$. One reason for this difference is the extremely high density (almost 200 fishes $/ 100 \mathrm{~m}^{2}$ ) of Anthiinae fishes (Serranids: Basslets) that we observed on submersible dives at Las Gemelas Seamount. These fish were distributed throughout all transects, as evidenced by the $100 \%$ occurrence on all dives. Threadfin Bass (Pronotogrammus multifascia$t u s$ ) and other Anthiinae fishes were commonly seen at Isla del Coco National Park (58.0\% and $41.7 \%$ of the dives, respectively), but occurred in smaller aggregations than at Las Gemelas Seamount. Higher densities of Scorpionfish were observed at Isla del Coco National Park than Las Gemelas, but Scorpionfish diversity was greater at Las Gemelas. Scorpionfish were present on every Las Gemelas dive, yet only on $75 \%$ of Isla del Coco National Park dives. Similarly, Eels were present on every Las Gemelas dive, but on only $50 \%$ of Isla del Coco National Park dives. Flagfins were relatively common at
Isla del Coco National Park, but this taxon was absent from the Las Gemelas dives.

Despite these differences, on a per-dive basis, there was no difference in biomass between the two sites when all species were lumped. The average biomass among dives at Las Gemelas $\left(348.3 \mathrm{~g} / \mathrm{m}^{2}\right)$ was not significantly different than the average biomass among dives at Cocos Island $\left(320.5 \mathrm{~g} / \mathrm{m}^{2}\right)$ in a two-sample t-test $(\mathrm{p}>0.05)$. There were differences, however, for species groups present at both locations. The biomass of Eels and the Anthias spp. complex was significantly greater at Las Gemelas than at Cocos Island in a twosample T-test $(\mathrm{p}<0.05)$. The biomasses of the rest of the species groups (groupers, scorpionfishes, wrasses, codlings, threadfin basses) were not significantly different between Las Gemelas and Cocos Island ( $p>0.05)$. These same patterns occurred in biomass; the standing stock of Basslets was $21.6 \mathrm{~kg} / 100 \mathrm{~m}^{2}$ at Las Gemelas Seamount and the Threadfin Bass comprised the largest component of biomass at 
Isla del Coco National Park $\left(10.18 \mathrm{~kg} / 100 \mathrm{~m}^{2}\right)$. We divided the biomass $\left(\mathrm{kg} / 100 \mathrm{~m}^{2}\right)$ of each taxonomic group observed at Las Gemelas Seamount by the biomass of each species at Isla del Coco National Park to develop a biomass ratio for the two sites (Table 5). The biomass ratio was greater at Las Gemelas than Isla del Coco National Park in four out of the seven categories of taxa that were present at both locations.

Size frequency of fishes: We were able to estimate the lengths of 2,040 fish (Table 6). In addition to being more abundant, Basslets were significantly larger (t-test, $\mathrm{p}<0.001$ ) at Las Gemelas Seamount than at Isla del Coco National Park. Conversely, Threadfin Bass, were more abundant and significantly larger (t-test, $\mathrm{p}<0.001)$ at Isla del Coco National Park than at Las Gemelas Seamount. As a group, Scorpionfishes were larger at Las Gemelas Seamount, but this is due to the presence of larger species of Scorpionfishes at that site.

Although not statistically significant (K-S test, $\mathrm{p}=0.313$ ), a plot of the percentage of total biomass by size class at each site indicates that larger fishes comprise a higher proportion of the biomass at Isla del Coco National Park than at Las Gemelas Seamount (Fig. 1). At Isla del Coco National Park, fishes greater than $50 \mathrm{~cm}$ long provide $28 \%$ of the total biomass, whereas at Las Gemelas, fishes longer than $50 \mathrm{~cm}$ comprise only $16 \%$ of the biomass. The difference is due to the lower numbers of medium-sized groupers and much larger numbers of small fishes at Las Gemelas Seamount. We observed the largest groupers on transects at the seamount, but relatively more groupers at Isla del Coco National Park. Also, the difference is caused by the densities of the Threadfin Bass and other Anthiinae fishes; the two most dominant taxa at each site. These small Serranids provide almost $80 \%$ of the biomass at Las Gemelas Seamount but only $44 \%$ of the biomass at Isla del Coco National Park.

Threadfin Bass and other Anthiinae fishes play the same role in the ecosystem (as predators of small fishes and prey of larger fishes such as groupers), and were stratified by depth,

TABLE 6

Mean length and SE of fishes observed on quantitative submersible transects

\begin{tabular}{|c|c|c|c|c|c|c|}
\hline \multirow{2}{*}{ Common Name } & \multicolumn{3}{|c|}{ Isla del Coco National Park } & \multicolumn{3}{|c|}{ Las GemelasSeamount } \\
\hline & Mean length (cm) & Number & SE & Mean length $(\mathrm{cm})$ & Number & $\mathrm{SE}$ \\
\hline Batfishes & 15.0 & 1 & & & & \\
\hline Brotulas & 30.0 & 1 & & & & \\
\hline Codlings & 18.5 & 23 & 1.0 & 16.3 & 4 & 2.4 \\
\hline Eels & 35.0 & 2 & 0.0 & 30.6 & 8 & 2.0 \\
\hline Flagfins & 25.9 & 184 & 0.5 & & & \\
\hline Flatfishes & 9.0 & 29 & 0.5 & & & \\
\hline Goosefishes & 15.0 & 1 & & 15.0 & 1 & \\
\hline Jellynose fish & 25.0 & 1 & & & & \\
\hline Leather bass & 30.0 & 4 & 0.0 & & & \\
\hline Longfinned bullseye & 57.5 & 2 & 2.5 & & & \\
\hline Ocean whitefish & 47.3 & 13 & 2.1 & & & \\
\hline Scorpionfishes & 17.6 & 111 & 0.7 & 27.4 & 17 & 2.4 \\
\hline Searobins & 9.1 & 16 & 0.5 & & & \\
\hline \multicolumn{7}{|l|}{ Serranids } \\
\hline Basslets & 15.5 & 143 & 0.4 & 18.6 & 637 & 0.2 \\
\hline Groupers & 75.0 & 2 & 5.0 & 85.0 & 2 & 5.0 \\
\hline Threadfin Bass & 20.8 & 619 & 0.1 & 19.0 & 215 & 0.3 \\
\hline Wrasses & 20.0 & 2 & 0.0 & 20.0 & 2 & 0.0 \\
\hline Total & & 1154 & & & 886 & \\
\hline
\end{tabular}




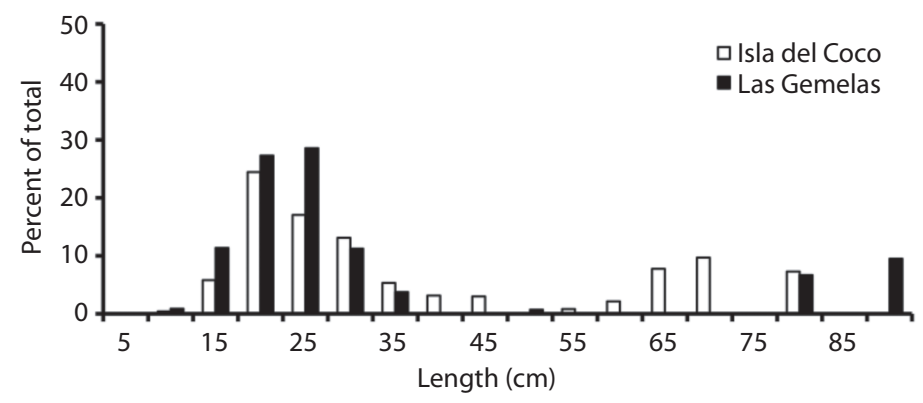

Fig. 1. The contribution of biomass of all species combined as a function of the size class of fishes observed at Isla del Coco National Park and Las Gemelas Seamount.

thus providing prey to larger fishes at a wide variety of depths (i.e., 150-300m). Threadfin Bass occurred at depths of 160-225m (Fig. 2 ), and were most often observed in large aggregations around large rock boulders, usually at depths of about 180-200 m. Other species of Basslets, however, occupied generally deeper depth zones, and were most frequently observed in or near the bottom, often lodged in cracks and crevices of rock habitats. The mean depth of other Basslets $(231 \mathrm{~m})$ was significantly different (t-test, $\mathrm{p}<0.001)$ than the mean depth of Threadfin Bass (181m). Larger groupers also were observed in aggregations of four to 100 fish. Less commonly we observed individual groupers near large boulders.

Species-Habitat associations: When density of fishes is viewed by habitat type, the combined density of fishes in habitats comprised primarily of bedrock or large boulders outcrops (RR and RB habitat categories) was significantly different (Chi Square, $\mathrm{p}<0.001$ ) between the two areas; density was more than three times as high at Las Gemelas Seamount as it was at Isla del Coco National Park (Fig. 3). This discrepancy was caused by the extremely high concentration of other Anthiinae fishes

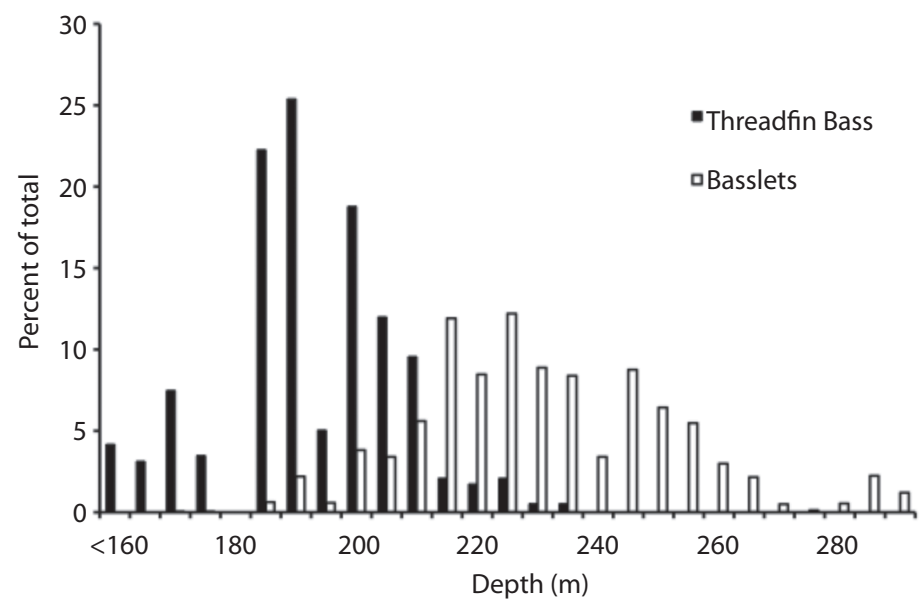

Fig. 2. Frequency histogram of observed depths for Threadfin bass (P. multifasciatus) $(\mathrm{n}=575)$ and all other Basslets (Anthiinae) $(\mathrm{n}=780)$. Data were used from all transects for which depth data were available at Isla del Coco National Park and Las Gemelas Seamount. 


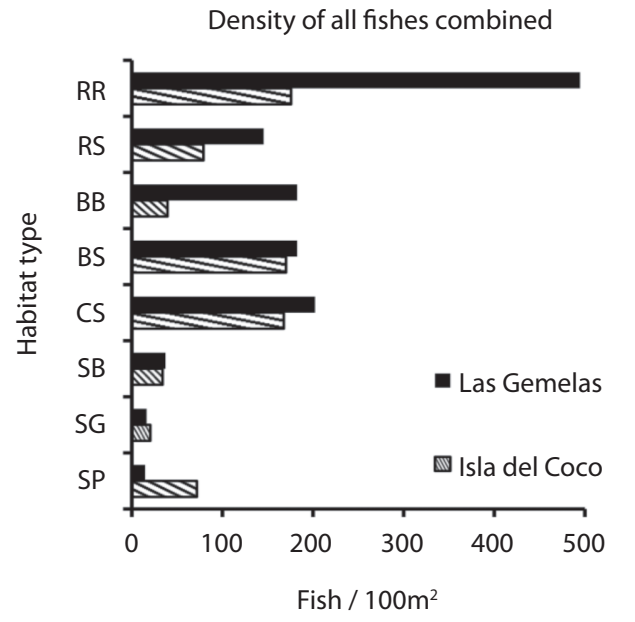

Fig. 3. Combined density $\left(\mathrm{Fish} / 100 \mathrm{~m}^{2}\right)$ of all fishes observed, by selected habitat types. Data were used from all quantitative transects for which depth data were available at Isla del Coco National Park and Las Gemelas Seamount. Habitat codes are based on the percentage of bottom covered by boulder (B), cobble (C), gravel (G), pebble $(\mathrm{P})$, bedrock or rock outcrop $(\mathrm{R})$, sand $(\mathrm{S})$, or vertical pinnacle $(\mathrm{T})$. The first letter represents the substrate type that accounted for at least $50 \%$ of the seafloor; the second code represents the substrate type that accounted for at least $20 \%$ of the seafloor.

in rocky habitats at Las Gemelas Seamount. Densities of fishes in the other habitats were similar between the two sites. Similarly, when estimates of fish density were plotted by slope categories (Fig. 4) the density was significantly different between the two areas (Chi Square, $\mathrm{p}<0.001$ ); density was much greater on steep slopes, which were usually comprised of rock habitats. Also, the density of fishes was significantly different between the two areas (Chi Square, $\mathrm{p}<0.001$ ); density was greatest on high rugosity habitats (Fig. 5). This is to be expected as the Anthiinae are adapted to avoid predation by taking refuge in holes and crevices throughout their range.

Occurrence of fishing gear: Lost fishing gear was observed on all submersible dives at Las Gemelas Seamount and on $50 \%$ of the dives at Isla del Coco National Park. On six submersible dives, discarded fishing line was

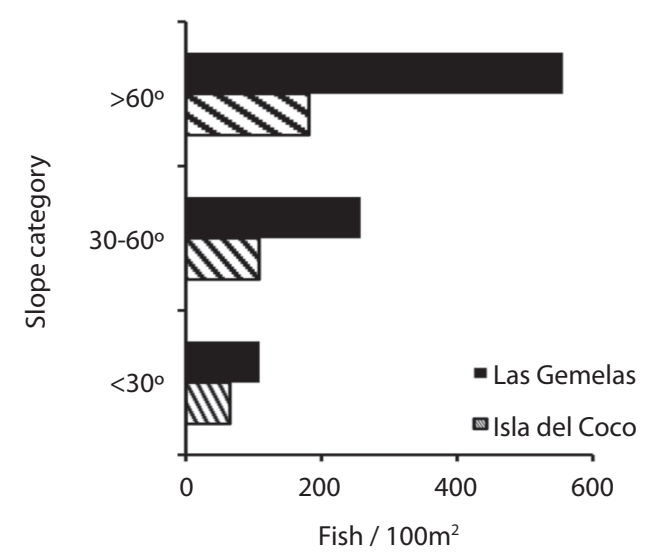

Fig. 4. Combined density $\left(\mathrm{Fish} / 100 \mathrm{~m}^{2}\right)$ of all fishes observed, by slope categories. Data were used from all quantitative transects for which depth data were available at Isla del Coco National Park and Las Gemelas Seamount.

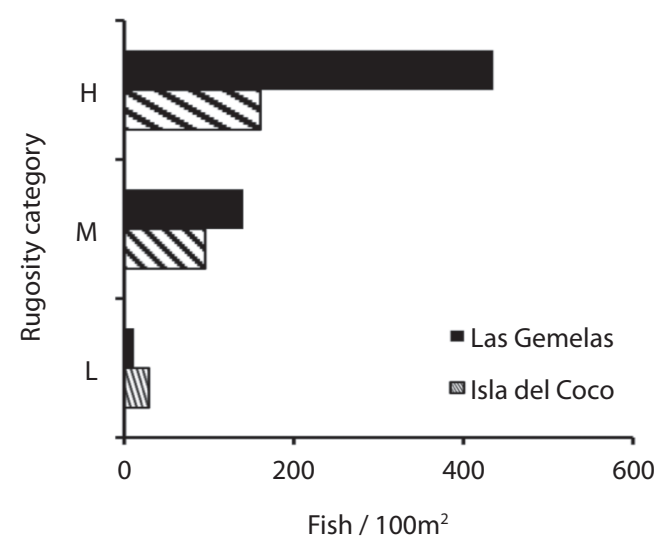

Fig. 5. Combined density $\left(\mathrm{Fish} / 100 \mathrm{~m}^{2}\right)$ of all fishes observed, by rugosity categories. Data were used from all quantitative transects for which depth data were available at Isla del Coco National Park and Las Gemelas Seamount. Rugosity levels (L: Low, M: Medium, H: High) are based on the capability of the crevices in the substrate to hide fishes.

noted on 33 occasions, during nine of the 38 quantitative transects. Aside from the observations of fishing line during quantitative transects on those dives, the presence of fishing line was noted by observers when the submersible was off transect on eight additional dives. Presence of fishing line was greatest at Las 
Gemelas Seamount; 30 of the 33 observations of fishing line occurred at Las Gemelas.

\section{DISCUSSION}

Seamounts are important features in the world's oceans, but until recently have been poorly studied (Clark et al. 2010). An increasing amount of research has been devoted to studying the biological communities and patterns of benthic biodiversity of seamounts. Not surprisingly, with increased information more questions are generated about the ecological role of seamounts and their vulnerability to disturbance. For example, Hubbs (1959) theorized that seamounts were important "islands" of biological endemism, a concept supported by Richer de Forges et al. (2000) who suggested that southwest Pacific seamounts contained greater endemism than that of deep-sea vent communities.

Although Samadi et al. (2006) indicated that their genetic studies in a relatively small area in the western Pacific might refute the concept of increased endemism on seamounts, they did agree with the hypothesis that seamounts are diversity hotspots, possessing benthic assemblages with particularly high species richness. Worm et al. (2003) provided a rationale for the increased relative abundance of organisms found on seamounts by suggesting that higher trophic level predators are found in higher diversity over seamounts because of currents that trap diurnally migrating plankton. Koslow et al. (2000) also described high densities of fishes associated with seamounts and discussed concerns about the vulnerability of seamount communities to human impacts, especially with the development of large-scale bottom trawl fisheries in the deep sea. The high densities of fishes and invertebrates in the area around Isla del Coco National Park and Las Gemelas seamount signifies the need for increased protection and study of these areas, because of the paucity of information related to the distribution and relative abundance of the important resources that could easily be overexploited.
The diversity of fish species in deep water at Isla del Coco National Park was lower than the diversity of fishes in shallow water in the National Park, and eight species groups accounted for more than $95 \%$ of the biomass of fishes. Two taxa, the Threadfin bass (Serranidae: Pronotogrammus multifasciatus) and other Anthiinae species, comprised $44 \%$ of the biomass at Isla del Coco National Park. Habitats surveyed at Isla del Coco National Park included vertical rock walls and steep slopes comprised of volcanic rock outcrops and sand. Habitats surrounding Isla del Coco National Park were often highly fragmented and contained many cracks and crevices for small fishes to hide. The edge of the shelf, at about $180-220 \mathrm{~m}$ deep contained the highest density of fishes; we often saw aggregations of hundreds of small fishes covering rock outcrops. These fishes in turn provide food for larger fishes such as groupers and sharks. In some areas we occasionally encountered large aggregations of groupers. These large aggregations were noted in our qualitative observations, but not included in quantitative assessments of groupers. The aggregations of groupers were some of the larger ones we have seen at any place in the world other than those in spawning aggregations.

The number of taxa observed in waters deeper than $50 \mathrm{~m}$ at Isla del Coco National Park and at Las Gemelas Seamount during our study was similar to that reported by Pearcy et al. (1989) and Tissot et al. (2007) for limited number of dives in deep rocky banks in the temperate waters of Oregon, USA, but far less than the 110 taxa reported by Starr and Yoklavich (2008) for extensive submersible surveys off California, USA. More submersible dives, covering more habitat types, will undoubtedly increase the list of known deepwater species near Isla del Coco National Park. Because Isla del Coco National Park and Las Gemelas Seamount are situated in the Eastern Tropical Pacific marine province (Robertson et al. 2004), we expect that many of the deepwater taxa reported to occur in the Galapagos Islands by Grove and Lavenberg (1997) would occur 
near Isla del Coco National Park. Although we expect similarities in genera, we expect different species as fishes from the same genus have proven to be different species near Isla del Coco National Park than at the Galapagos.

Although species richness was higher at Isla del Coco National Park (due to the increased area surveyed there), the density of fishes, both in terms of numbers and biomass per unit area, was greater at Las Gemelas Seamount. Habitats we surveyed at Las Gemelas contained rich and dense communities of invertebrates and fishes. Importantly, we encountered different habitats in each of our dives at Las Gemelas, indicating that the seamount is likely to include a wider variety of habitats than we surveyed. This suggests that the diversity of species at Las Gemelas Seamount is potentially much greater than we were able to determine with the available submersible dives. Even with the small sample size at Las Gemelas Seamount, however, our data support the hypothesis that density of fishes in this region is high. The densities of 138 and 253 fishes $/ 100 \mathrm{~m}^{2}$ are similar to those observed in eastern-Pacific temperate waters. The density of fishes (excluding young-of-theyear fish) estimated from submersible surveys in water depths of 50-375m in waters off California and Oregon, USA, has been reported as ranging from $40-225$ fishes $/ 100 \mathrm{~m}^{2}$ (Yoklavich et al. 2002), about $70 \mathrm{fish} / 100 \mathrm{~m}^{2}$ (Tissot et al. 2007), and $65-185$ fishes $/ 100 \mathrm{~m}^{2}$ (Starr \& Yoklavich 2008). In the small sample size we have, we speculate that the deeper waters off Isla del Coco National Park are less diverse, but have a higher fish density than other locations because of the extraordinarily high biomass of Anthiinae species we observed.

In terms of fish diversity and abundance, Las Gemelas Seamount probably have a similar diversity of fishes as the deep water off Isla del Coco National Park, and overall contain a higher biomass of fishes. Importantly, however, Las Gemelas Seamount contained a much lower abundance of large predatory fishes. On each dive, we observed large densities of fishes $20 \mathrm{~cm}$ long or less, but relatively few large predators, such as groupers, which would be expected to feed on the large biomass of small fishes. The groupers we did see at Las Gemelas Seamount were larger than those observed at Isla del Coco National Park. Anecdotes provided by local fishermen indicate that historically, 1000 groupers a day were caught at certain times of the year by a group of 20 fishing boats using hook and line fishing gear at the seamount. Given these historic densities, we expected to observe many more groupers at Las Gemelas Seamount. The relatively low numbers of groupers we saw may be due to a low sample size (number of dives), the fact that the fishing occurred on spawning aggregations and our surveys occurred at a different time of year, or a result of the intense fishing pressure that has occurred at Las Gemelas Seamount. Conversely, the extremely high density of Anthiinae species at Las Gemelas may be due to the low numbers of large predatory fishes at Las Gemelas Seamount.

Given the diverse and rich assemblages of invertebrates carpeting the bottom habitats at Las Gemelas Seamount (we saw fishing line, but no evidence of alteration of invertebrate communities from fishing activities), and the extremely high numbers of Basslets, which are prey items of larger groupers, we expect that the Las Gemelas Seamount are a prime candidate for increased protection. Without fishing pressure, we would expect the numbers of large groupers to increase, given the linkages between relatively pristine benthic habitats, the presence of large numbers of prey fishes, and grouper populations. Another reason for considering increased protection for Las Gemelas Seamount is that we are confident that the area contains species that have not yet been described in the scientific literature, and thus are important for the maintenance of biodiversity. We have consulted with world experts in the taxonomy of Anthiinae, and although we will not know for certain until we obtain voucher specimens, we believe that we encountered at least one new species of Anthiinae at Las Gemelas Seamount. The same may also be true for several species of Batfishes and 
Scorpionfishes that we observed during our submersible surveys.

The observed differences between the two survey locations may be due to the greater depth range and variety of habitats surveyed at Isla del Coco National Park, the larger number of transects conducted at Isla del Coco National Park, or it may be an effect of the island biogeography typical of tropical islands. Until more surveys are conducted to enable an analysis of species-area curves, it is not possible to determine the reason for the observed differences. One important qualitative observation is that we saw a larger number of encrusting and structure-forming invertebrates at Las Gemelas Seamount (See Starr et al. Revista de Biología Tropical, this issue); this very rich invertebrate community composition indicates that habitats at Las Gemelas may be able to harbor a greater diversity and biomass of fishes than at Isla del Coco National Park.

Given that the island groups in the Tropical Eastern Pacific (i.e., Galapagos, Malpelo, Isla del Coco) are known for endemic species (Grove and Lavenberg 1997, Garrison 2005), we expect that the seamount communities near the Tropical Eastern Pacific island groups would also be reflective of ecologically isolated communities. This highlights the need for increased study of the biological communities at Las Gemelas Seamount - to determine the role of the seamount in maintenance of marine biodiversity.

\section{ACKNOWLEDGMENTS}

We thank Odalisca Breedy, Jorge Cortés, Shmulik Blum, Sylvia Earle, Avi Klapfer, Bruce Robison, Edith Widder, and the crew of the Argo (Undersea Hunter Group) for safe submersible operations and help with observations of fishes and macroinvertebrates. We thank John McCosker, Bob Lea, Douglas Long, Matt Craig, Rachel Arnold, and Gregor Cailliet for help with identifying species from photographs. Cheryl Barnes provided some statistical analysis and helped finalize the figures and tables. Comments from three anonymous reviewers greatly improved the manuscript. Funding was provided by the National Geographic Society, the Walton Family Foundation, the Waitt Foundation, California Sea Grant Program, Moss Landing Marine Labs, and the University of Costa Rica.

\section{RESUMEN}

Las faunas de aguas profundas de islas oceánicas del Pacífico Tropical Oriental se conocen poco y de los montes submarinos nada. Del 11 al 22 de septiembre de 2009 llevamos a cabo una exploración de zonas profundos del Área de Conservación Marina Isla del Coco utilizando un submarino tripulado. El objetivo del estudio fue caracterizar los hábitats y las comunidades, y cuantificar las poblaciones de peces de profundidad en la Isla del Coco y los montes submarinos Las Gemelas, situados a $50 \mathrm{~km}$ al suroeste de la Isla del Coco. Realizamos 22 inmersiones con el submarino, con más de 80 horas de observación submarina, y filmamos 30 horas de video. Investigamos hábitats entre $50-402 \mathrm{~m}$ de profundidad y observamos más de 45 especies de peces, algunas de las cuales son especies nuevas para la ciencia. La diversidad de peces profundos en la Isla del Coco fue menor que en aguas someras, y ocho grupos de especies representaron más del 95\% de la biomasa total de peces. La densidad combinada de peces fue 253 peces $/ 100 \mathrm{~m}^{2}$ en Las Gemelas y 138 peces $/ 100 \mathrm{~m}^{2}$ en la Isla del Coco.

Palabras clave: Isla del Coco, peces de profundidad, observaciones desde submergible, biodiversidad.

\section{REFERENCES}

Alvarado, G.E. 2009. Los volcanes de Costa Rica: Geología, historia, riqueza natural y su gente, 3rd ed. EUNED, San José, Costa Rica. 335 p.

Anderson, W.D. Jr. \& C.C. Baldwin. 2000. A new species of Anthias (Teleostei: Serranidae: Anthiinae) from the Galápagos Islands, with keys to Anthias and eastern Pacific Anthiinae. Proc. Biol. Soc. Washington 113: 369-385.

Béarez, P. \& P. Jiménez-Prado. 2003. New records of serranids (Perciformes) from the continental shelf of Ecuador with a key to the species, and comments on ENSO-associated fish dispersal. Cybium 27: 107-115.

Clark, M.R., A.A. Rowden, T. Schlacher, A. Williams, M. Consalvey, K.I. Stocks, A.D. Rogers, T.D. O’Hara, M. White, T.M. Shank \& J.M. Hall-Spencer. 2010. The Ecology of Seamounts: Structure, Function, and Human Impacts. Annu. Rev. Mar. Sci. 2: 253-278. 
Cortés, J. 2008. Historia de la investigación marina de la Isla del Coco, Costa Rica. Rev. Biol. Trop. 56 (Suppl. 2): 1-18.

Cortés, J. \& A. Blum. 2008. Life to $450 \mathrm{~m}$ depth at Isla del Coco, Costa Rica. Rev. Biol. Trop. 56 (Suppl. 2): 189-206.

Froese, R. \& D. Pauly (Eds.). 2012. FishBase. World Wide Web electronic publication. www.fishbase.org, version (04/2012).

Garrison, G. 2005. Peces de la Isla del Coco/Isla del Coco Fishes, 2nd ed. Edit. INBio, Heredia, Costa Rica. $429 \mathrm{p}$

Greene, H.G., M.M. Yoklavich, R.M. Starr, V.M. O'Connell, W.W. Wakefield, D.E. Sullivan, J.E. McRea \& G.M. Cailliet. 1999. A classification scheme for deep seafloor habitats. Oceanol. Acta 22: 663-678.

Grove, J.S. \& R.J. Lavenberg. 1997. The Fishes of the Galápagos Islands. Stanford Univ. Press, Stanford, California, 863 p.

Hubbs, C.L. 1959. Initial discoveries of fish faunas on seamounts and offshore banks in the eastern Pacific. Pac. Sci. 12: 311-316

Jiménez-Prado, P. \& P. Béarez. 2004. Peces marinos del Ecuador continental. Tomo 2: Guía de Especies / Marine Fishes of Continental Ecuador. Volume 2: Species Guide. SIMBIOE/NAZCA/IFEA.

Koslow J.A., G.W. Boehlert, J.D.M. Gordon, R.L. Haedrich, P. Lorance \& N. Parin. 2000. Continental slope and deep-sea fisheries: Implications for a fragile ecosystem. ICES J. Mar. Sci. 57: 548-557

Lizano, O.G. 2008. Dinámica de aguas alrededor de la Isla del Coco, Costa Rica. Rev. Biol. Trop. 56 (Suppl. 2): $31-48$.

McCosker, J.E. 2008. Trachyscorpia osheri and Idiastion hageyi, two new species of deepwater Scorpionfishes (Scorpaeniformes: Sebastidae, Scorpaenidae) from the Galápagos Islands. Proc. California Acad. Sci. 59: 113-123.

Poss, S.G. 1995. Scorpaenidae. Rascacios, chancharros. p. 1544-1564. In W. Fischer, F. Krupp, W. Schneider, C. Sommer, K.E. Carpenter and V. Niem (eds.) Guía FAO para identification de especies para lo fines de la pesca. Pacífico Centro-Oriental. 3 Vols. FAO, Rome.

Poss, S.G., J.E. McCosker \& C.C. Baldwin. 2010. A new species of Scorpaenodes (Pisces: Scorpaenidae) from the Galápados and Cocos Islands with discussions of the limits of Scorpaenodes and Thysanichthys. Proc. California Acad. Sci. 61: 235-267.
Pearcy, W.G., D.L. Stein, M.A. Hixon, E.K. Pikitch, W.H. Barss \& R.M. Starr. 1989. Submersible observations of deep-reef fishes of Heceta Bank, Oregon. Fish. Bull. 87: 955-965.

Richer de Forges, B., JA. Koslow \& G.C.B. Poore. 2000. Diversity and endemism of the benthic seamount fauna in the southwest Pacific Nature 405: 944-947.

Robertson, D.R., J.S. Grove \& J.E. McCosker. 2004. Tropical transpacific shore fishes. Pac. Sci. 4: 507-565.

Samadi, S., L. Bottan, E. Macpherson, B. Richer De Forges \& M.Boisselier. 2006. Seamount endemism questioned by the geographic distribution and population genetic structure of marine invertebrates. Mar. Biol. 149: 1463-1475.

SINAC, MINAET. 2008. GRUAS II: Propuesta de Ordenamiento Territorial para la conservación de la biodiversidad de Costa Rica. Volumen 3: Análisis de Vacíos en la Representatividad e Integridad de la biodiversidad marina y costera. Sistema Nacional de Áreas de Conservación (SINAC) del Ministerio de Ambiente, Energía y Telecomunicaciones (MINAET), San José, Costa Rica. 60 p.

Starr, R.M. \& M.M. Yoklavich. 2008. Monitoring MPAs in Deep Water off Central California: 2007 IMPACT Submersible Baseline Survey. California Sea Grant College Program Publ. T-067. 24 pp.

Starr, R.M., D.S. Fox, M.A. Hixon, B.N. Tissot, G.E. Johnson \& W.H. Barss. 1996. Comparison of submersible-survey and hydroacoustic-survey estimates of fish density on a rocky bank. Fish. Bull. 94: 113-123.

Stein, D.L., B.N. Tissot, M.A. Hixon \& W. Barss. 1992. Fish-habitat associations on a deep reef at the edge of the Oregon continental shelf. Fish. Bull. 90: 540-551.

Tissot, B.N., M.A. Hixon \& D.L. Stein. 2007. Habitatbased submersible assessment of macro-invertebrate and groundfish assemblages at Heceta Bank, Oregon, from 1988 to 1990. J. Exp. Mar. Biol. Ecol. 352: 50-64.

Worm, B., H.K. Lotze \& R.A. Myers. 2003. Predator diversity hotspots in the blue ocean. Proc. Natl. Acad. Sci. USA 100: 9884-9888.

Yoklavich, M.M., G.M. Cailliet, R.N. Lea, H.G. Greene, R. Starr, J. deMarignac \& J. Field. 2002. Deepwater habitat and fish resources associated with the Big Creek Ecological Reserve. California Coop. Ocean. Fish. Investig. Rep. 43: 120-140.

Zar, H. 1999. Biostatistical Analysis, 4th Ed. Prentice Hall, Upper Saddle River, New Jersey. 